\title{
Segmentation and reconstruction of the 3D geometry of the middle and inner ear
}

\author{
Yanfei $\mathrm{Lu}^{1, *}$ \\ ${ }^{1}$ Warsaw University of Technology, Faculty of Production Engineering, Institute of Mechanics and Printing, Department of \\ Construction Engineering and Biomedical Engineering, Narbutta 85, 02-524 Warsaw, Poland
}

\begin{abstract}
The anatomical model of the ear is of great importance in the design of ossicular prosthesis, cochlear implant electrodes, as well as for the preoperative planning and navigation of surgery. By means of micro-computed tomography (micro-CT) and technology of 3D reconstruction, an anatomical model of the middle and inner ear was built. Region of interest includes the ossicular chain (malleus, incus, and stapes), cochlea (scala vestibule-ST, scala tympaniST, basilar membrane-BM, spiral ligament-SL and osseous spiral lamina-OSL), tympanic membrane-TM, oval window membrane-OWM, round window membrane-OWM and stapedial annular ligament-SAL. The micro-CT images of a cadaver's temporal bone were acquired by "SkyScan 1076" (Kontich, Belgium, www.skyscan.be) and then reconstructed to cross-section images by SkyScan NRecon ${ }^{\mathrm{TM}}$ (v1.6.10.4). The image processing and 3D geometry reconstruction of temporal bone were performed by software Mimics ${ }^{\circledR}$ (v14.0, Materialise NV, Leuven, Belgium). The obtained structures are measured and validated against literature data and the results are in good agreement.
\end{abstract}

\section{Introduction}

Middle ear and inner ear are important structures responsible for sound transduction and balance. Sound wave in the air is firstly detected by eardrum (tympanic membrane), then transformed through ossicular chain to cochlea and there transduced to bioelectric signal. Ossicular chain in the middle ear consists of malleus, incus and stapes. The three ossicles are attached to surrounding bony structures by ligaments and tendons and are connected with each other by two joints. In this way, they keep a complex spatial structure and fulfill the function of sound transmission. Malleus connects to tympanic membrane, and stapes connects to cochlea. Around the footplate of stapes, there is a ring of fibrous tissue that connects the stapes to the oval window of the inner ear, called stapedial annular ligament. The opening on the cochlea in contact with stapes is covered with a membrane, called oval window membrane.

The bony labyrinth of the inner ear is the network of passages with bony walls lined with periosteum in the temporal bone. It consists of three parts: the vestibule, semicircular canals, and cochlea. Inside these cavities, there exists a system of endolymph filled tube, membranous labyrinth, which is separated from bony labyrinth by a layer of perilymph fluid. Cochlea is a spiral shaped cavity in the bony labyrinth. In humans, it makes about 2.5 turns around its axis. There are three scalae in the cochlea: scala vestibule, scala tympani and scala media. Scala vestibule lies superior to the cochlea spiral and filled with the fluid of perilymph. Scala tympani lies inferior to cochlea spiral and filled with the fluid of endolymph. Between them, there is scala media containing also the fluid of endolymph. Normally, the membrane between SV and SM, Reissner's membrane, is very thin and hard to illustrated by means of micro-CT. The basilar membrane, which separates apart the ST and SM, has some degrees of thickness that can be detected by micro-CT. Mechanical waves excited by the OWM, which locates on the bottom end of the SV, transfer along the spiral coli to the apex of the cochlea, the helicotrema, where the SV and ST merge, then come back along another duct ST. Sensory hair cells on the $\mathrm{BM}$ are activated by the fluid wave in SV and ST, and convert the mechanical signal to biological signal transferred to the brain along the auditory nerve. The other two parts of the bony labyrinth, the vestibule and semicircular canals, are responsible for the sensations of balance and motion. They use the same kinds of lymph fluids and sensory cells as the cochlea uses, and detect the attitude and motion of the body.

Diseases or injuries of the middle ear or inner ear may cause severe healing loss. About 5\% population suffer from disability associated with hearing problem worldwide [1]. The causes of hearing loss are different from conductive (middle ear) or sensorineural (inner ear). Ossicles prosthesis are applied to middle ear damage caused by chronic inflammatory or disease, such as otosclerosis (stiffness of stapes), to reconstruct the

\footnotetext{
Corresponding author: y.lu@,wip.pw.edu.pl
} 
conduction system [2, 3]. While damage of inner ear requires the application of cochlear implant.

Optimal designed ossicular prosthesis and cochlear implant electrodes, as well as their precise insertion are possible thanks to the development of computer-aided engineering (CAE) and computer-aided surgery (CAS): design of the ossicular prosthesis and cochlear implant electrodes is greatly simplified by computer-aided design (CAD) $[4,5]$; afterwards verification and optimization of them are achieved by finite element method (FEM) $[6,7$, 10]; due to the complexity and small scale of the structure of the middle and the inner ear, CAS is usually applied in preoperative surgery planning and navigation during the surgery $[8,9]$.

Effectiveness of these computer-based approaches is based on a reliable geometric model. To obtain the $3 \mathrm{D}$ anatomical information of the analyzed structures, several methods have been used, among them histological microgrinding [11], Magnetic Resonance Imaging (MRI) [12] and micro computed tomography (micro-CT). Histological microgrinding traditionally used and features for high accuracy but destructive procedure. MRI features for large contract for soft tissue but low spatial resolution. Therefor micro-CT is preferred for its high resolution and nondestructive procedure.

Aim of this work is to build a $3 \mathrm{D}$ anatomical model of the middle and inner ear by means of micro-CT and $3 \mathrm{~d}$ reconstruction. Region of interest includes the ossicular chain (malleus, incus, and stapes), cochlea (scala vestibule-ST, scala tympani-ST, basilar membrane-BM, spiral ligament-SL and osseous spiral lamina-OSL), tympanic membrane-TM, oval window membrane-OWM, round window membrane-OWM and stapedial annular ligament-SAL.

\section{Methods}

\subsection{Temporal bone preparation}

The human cadaver's temporal bone was dissected at Warsaw Medical University from male cadaver. The temporal bone was harvested within 2 days of death and removed from soft tissues and frozen immediately. The specimen was cut to the size about $2 \mathrm{~cm} \times 2 \mathrm{~cm} \times 2 \mathrm{~cm}$ cubic, in order to minimize the X-ray absorption by surrounding bone.

\subsection{Micro-CT scanning process and the image acquisition}

\subsubsection{Methodology of micro-CT scanning process}

Micro-CT is a non-invasive and efficient technique to investigate the $3 \mathrm{D}$ geometry of anatomic structure. In this study, to examine the structure of the middle and inner ear, a piece of extracted calvarial specimen was scanned using a micro-CT instrument "SkyScan 1076" (Kontich, Belgium, www.skyscan.be).
The micro-CT system consists of the X-ray lamp with a high voltage power supply, one bed for the sample with a precise object positioning mechanism and a CCD camera connected to a computer with software for storing and processing of the received data. This equipment is able to emit X-ray on energy range from $20 \mathrm{kV}$ to $100 \mathrm{kV}$. The data are obtained by rotating the $\mathrm{X}$-ray tube and the CCD cameras for up to 180 degrees or 360 degrees, with adjustable increments (at least 0.02 degree) around the axis of the immovable object. The resulting individual $2 \mathrm{D}$ projection $\mathrm{x}$-ray images are saved as 16-bit TIFF files. The rotation of the imaged sample relative to the $\mathrm{x}$-ray source and camera allows the precise $3 \mathrm{D}$ location of the scanned objects to be calculated.

\subsubsection{Parameters of the scanning process}

The scan was performed with a source voltage and source current of, respectively, $100 \mathrm{kV}$ and $100 \mu \mathrm{A}$. An aluminum filter with a thickness of $1 \mathrm{~mm}$ was used to narrow the X-ray energy spectrum, reducing the low energy component, thus reducing beam hardening. The CT images were acquired at a step angle of $0.6^{\circ}$ for a total circular orbit of $360^{\circ}$. Exposure time per frame was $620 \mathrm{~ms}$. In order to improve quality of the final image file, averaging of four images was conducted. Scanning resolution in this study is set at $18 \mu \mathrm{m}$. All projection images were saved as 16-bit TIFF files (Fig.1).

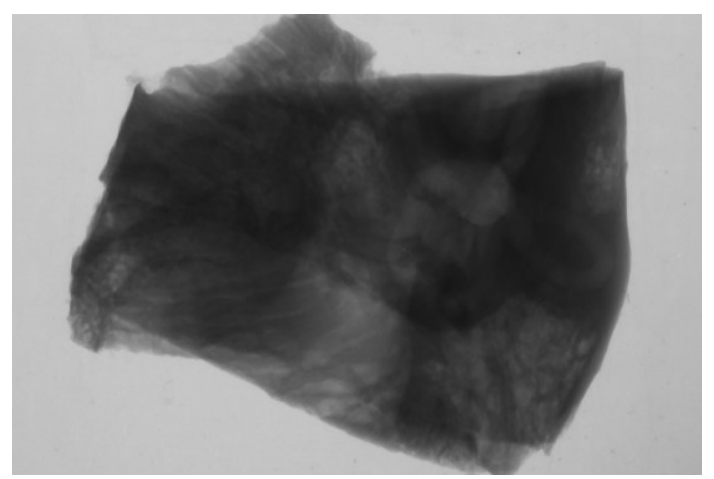

Fig. 1. Example 2D projection image of micro-CT scan.

\subsubsection{D Reconstruction}

Following projection images acquisition from multiple angles, cross-section reconstruction of from CT image was performed based on the Feldkamp algorithm which consists of back-projection and convolution. Backprojection derives object position from the shadow position from multiple angles, while convolution removes a blurring halo from around the periphery of objects which back-projection alone would leave, by a selective image subtraction method. In this study, SkyScan NRecon (Version 1.6.10.4) package was used to reconstruct cross-section images of the object from tomography projection.

Some imaging artifacts are peculiar to CT, such as ring artifacts and beam hardening. Beam hardening rises 
from emission from the micro-focus $\mathrm{x}$-ray source of a mixture of low and high energy x-rays. Ring artifacts arise from the incremental rotation of the object-camera geometry, in combination with non-uniformity of the $\mathrm{x}$-ray detection performance of individual camera pixels. During the reconstruction, these artifacts could be corrected by software to some extent.

The final projection images in 16-bit TIFF format were loaded and then the following parameters of the reconstruction process were adjusted by a try-and-error fashion and defined as: Ring Artifact Correction=10, Beam Hardening Correction $(\%)=17$. Post-alignment was set as 5.5 to reduce tails, doubling or blurring in the reconstructed images.

Region-of-interest was chosen to include the ossicular chain (malleus, incus, stapes), tympanic membrane and bony labyrinth (vestibule, semicircular canals and cochlea). Grey level threshold was adjusted according to a preview slice to make sure the structures of interest obvious and in good contrast. A total number of 600 slice images were saved in 8-bit grayscale BMP format at the resolution $2000 \times 1336$ pixels. Figure 2 shows an example of one slice image of the inner ear.

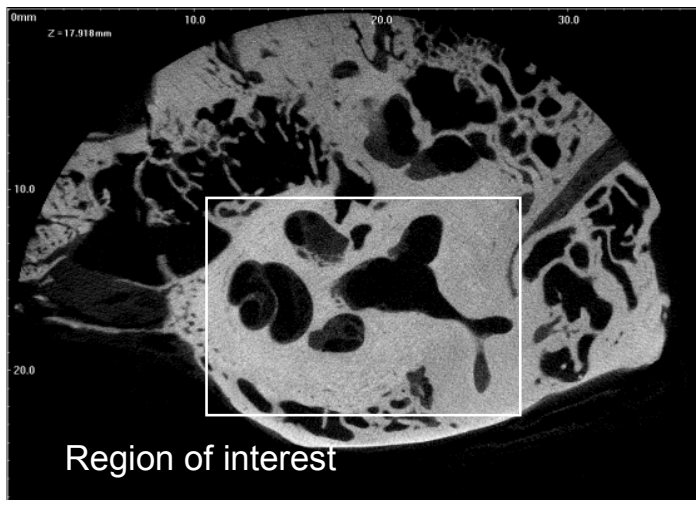

Fig. 2. Example of cross sectional image.

\subsection{Image processing and three-dimensional reconstruction}

In order to separate the regions of interest from the rest of the image, a series of segmentation tools include Thresholding, Region growing, Boolean and Morphology operations were used.

The first step was a separation of bone from soft tissue by Thresholding tool. The defined radiodensity for bone is from 226 to $3024 \mathrm{HU}$ and for soft tissue is from 700 to $225 \mathrm{HU}$. This defined CT value well reflected the bone and soft tissue partition, despite some of the unavoidable noise points which could be removed manually in later steps. Because the ossicular chain is connected to temporal bone only by ligaments and tenders which were previously removed in thresholding step, we could separate it to a new mask by Region growing tool. However, there are some structures cannot be separated by automatic tools: structures in between small gap such as the 2 joints between ossicles, SAL and stapes; structures have similar contrast such as BM, SL and OSL; thin layers such as RWM and OWM. In this case, manually segmentation is applied.

\section{Results}

Due to the small space between incus-malleus joint and incus-stapes joint, the separation of the ossicular chain was performed manually by Mask edit tool. After segmentation, we got three masks of Malleus, Incus and Stapes, as shown in Fig.3.

Closely next to malleus, there is a membranous structure-tympanic membrane (TM), which is marked in light green, as shown in Fig.4. The 3D view reveals the topological relationship between TM and the ossicular chain. The head of malleus attaches to the incus; and the handle of malleus is connected to the cone-shaped membrane TM.

Due to the big contrast between bone and soft tissue, it's not too difficult to fill the bony labyrinth. Spiral structure of cochlear and spatial arrangement of three semicircular canals could be clearly observed in Fig.5. Round window niche and oval window niche could also be noticed.

The next step was cut out of scala vestibuli and scala tympani from the mask of cochlear capsule. Scala vestibuli (SV) lies superior to the cochlea spiral and scala tympani (ST) lies to the inferior. Both of the two spiral-shaped canals make about 2.5 turns about the modiolus. Between the ducts of SV and ST is scala media. Organ of corti (OC) and basilar membrane (BM) were not marked separately in this project because thickness of BM $(5-13 \mu \mathrm{m})$ is less than 1 pixel $(18 \mu \mathrm{m})$. SV and ST join at the helicotrema, the tip of the helix.

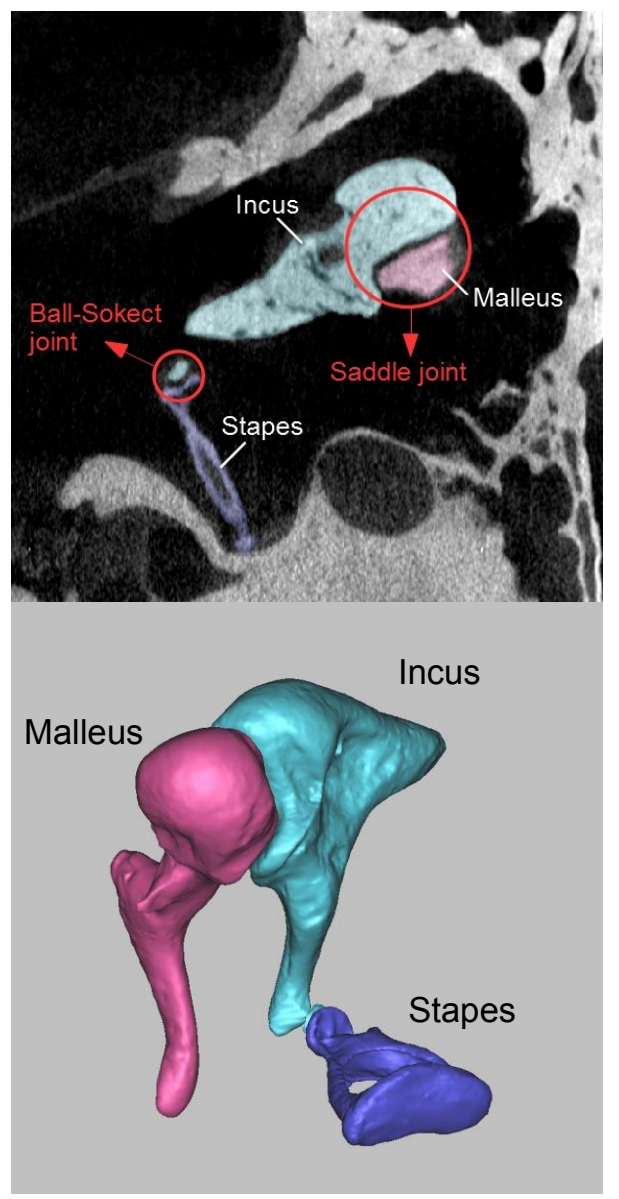

Fig. 3. Segmentation and 3D representation of ossicular chain. 


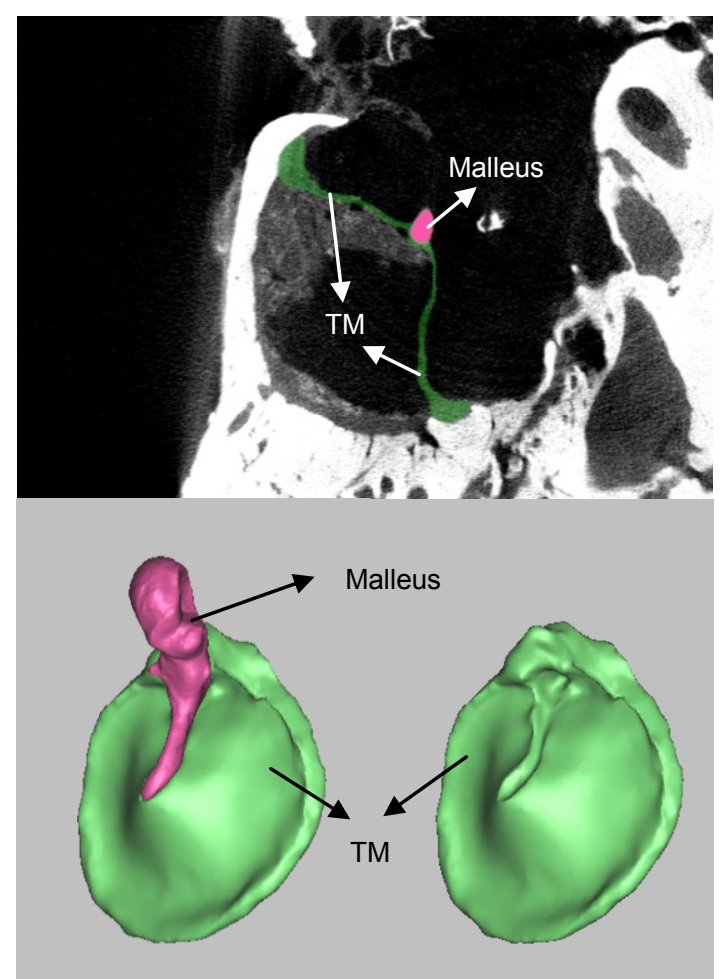

Fig. 4. Tympanic membrane (TM).

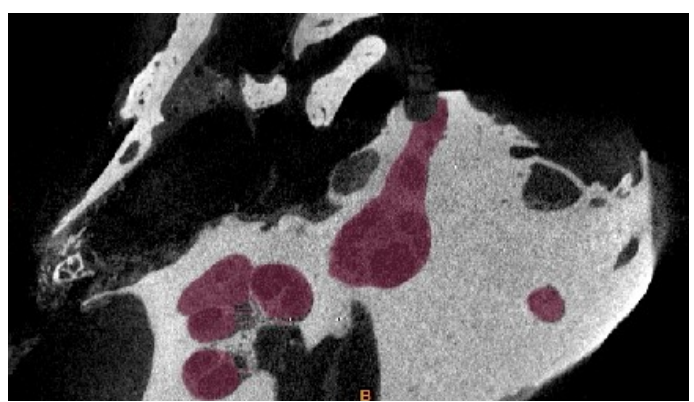

Fig. 5. Bony labyrinth.

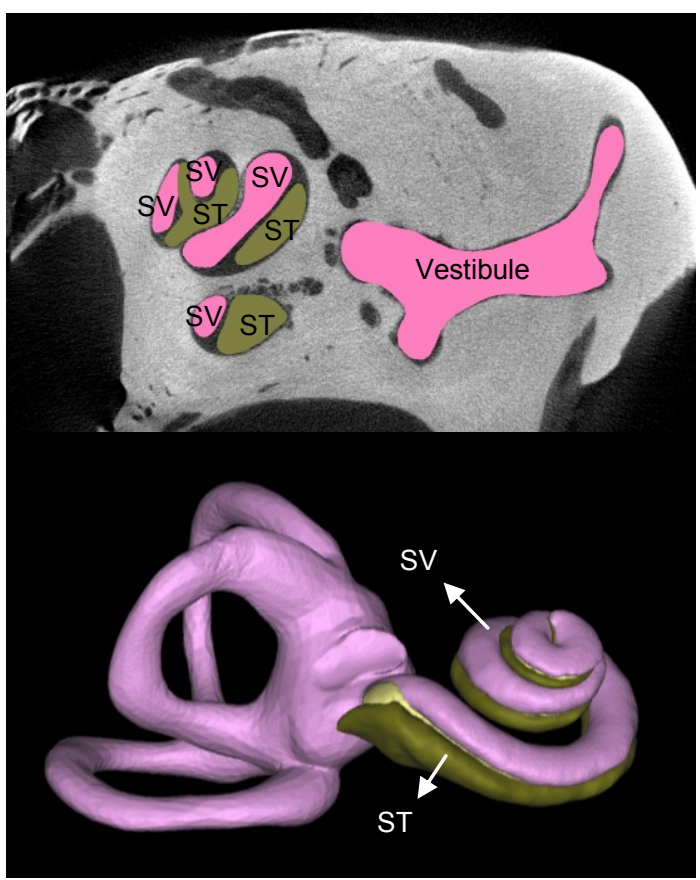

Fig. 6. Segmentation and 3D representation of scala vestibuli and scala tympani.

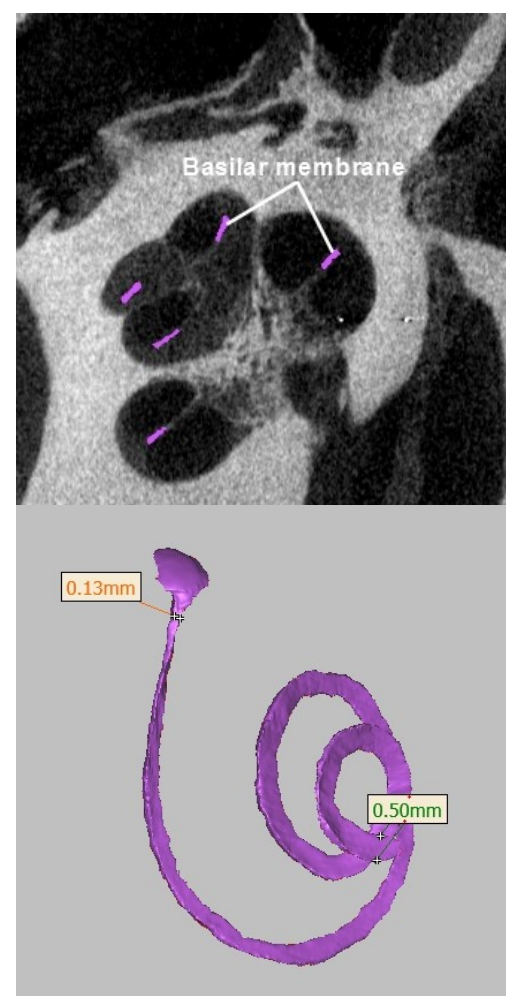

Fig.7. Basilar membrane and measurement.

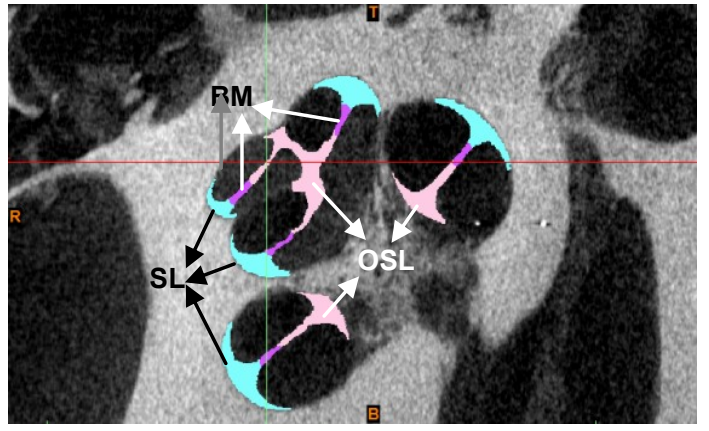

Fig.8. Segmentation of basilar membrane, spiral ligament and osseous spiral lamina.

In Fig.6, the gap between SV and ST, consisted of three parts, that are the compose of OC and BM, spiral ligament (SL) and osseous spiral lamina (OSL). Contrast between those parts was not big enough to separate them directly, therefore data reported in literature is referred, that width of $\mathrm{BM}$ is between $0.1 \mathrm{~mm}$ and $0.5 \mathrm{~mm}$, narrowest at the base and widest at the apex of cochlear. Mask and reconstruction of the compose of organ of corti and basilar membrane is shown in Fig.7. SL and OSL were obtained by subtracting BM, SV and ST from the mask of bony labyrinth, as shown in Fig.8.

Round window situated at the bottom end of ST, is a membrane-covered opening between the middle ear and the cochlea. Light mask represents the RWM. This saddle-shaped membrane seals the RW niche, as shown in Fig.9.

Another tiny structure of interest is stapedial annular ligament (SAL). SAL is a ring of fibrous tissue between the base of the stapes to the oval window bony wall, which allows the vibration of stapes and seals the oval window niche. Geometry of SAL is various, the average 
height is $0.2 \mathrm{~mm}$ and thickness is between 0.04 and 0.1 $\mathrm{mm}$. Mask and 3D reconstruction of SAL are shown as in Fig. 10.
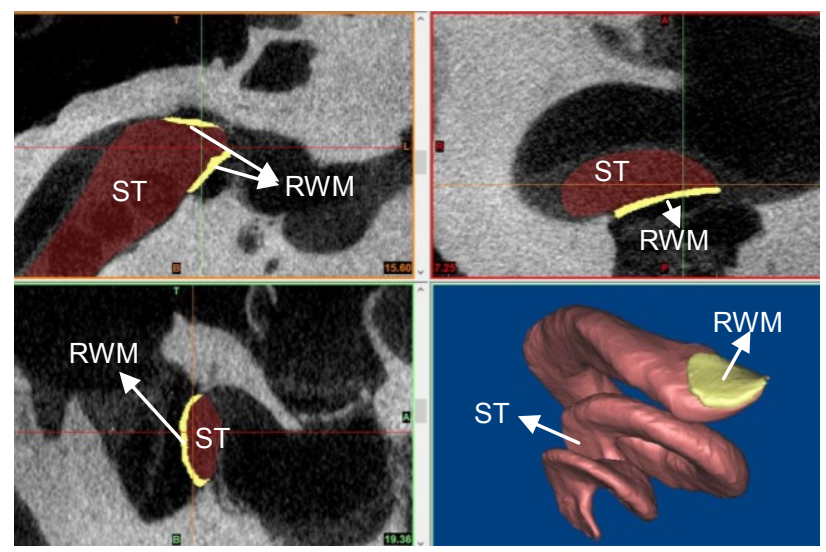

Fig.9. Segmentation of the round window membrane.

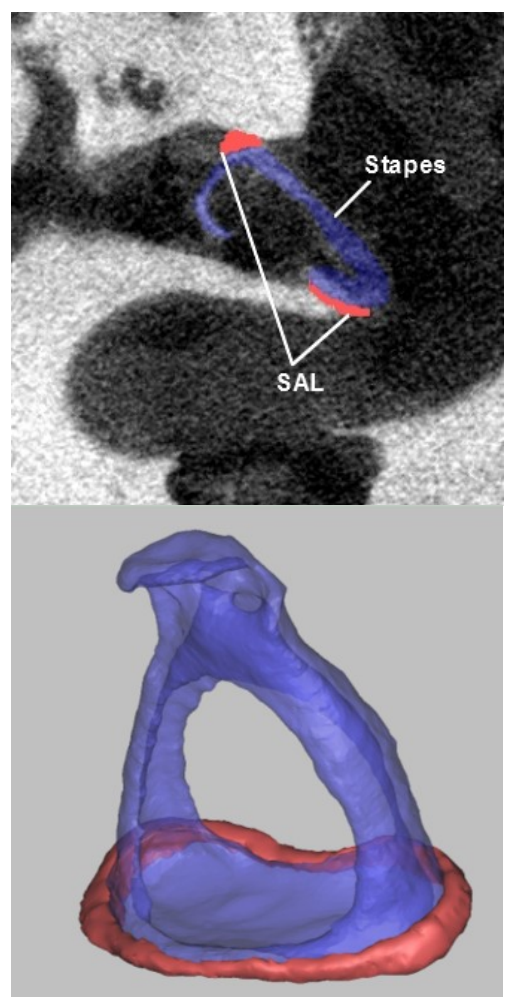

Fig.10. Segmentation of the stapes and the stapedial annular ligament.

Comparison of feature diameters are performed in order to verify the reliability of the 3D model. Firstly, the widths of 3 cochlea turns are measured and compared with data reported by Erixon [13]. The width of the first turn of cochlea is $8.17 \mathrm{~mm}$ (range 5.6-8.2 $\mathrm{mm}$, mean width $6.8 \mathrm{~mm}$ ), the width of the second turn is 4.25 (range $3.3-4.3 \mathrm{~mm}$, mean width $3.8 \mathrm{~mm}$ ) and the width of the third turn is $3.11 \mathrm{~mm}$ (range 0.6-3.6 mm, mean width $2.1 \mathrm{~mm}$ ). Semimajor anteroposterior and semiminor supero-inferior axes of stapes footplate measured in this 3D model are $2.99 \mathrm{~mm}$ and $1.80 \mathrm{~mm}$ respectively. In vitro measurement gives the results of $2.93 \mathrm{~mm}$ and $1.82 \mathrm{~mm}$ [14]. Measurement difference between this model and in vitro experiment are $2.0 \%$ and
$1.1 \%$ respectively, which are not larger than the error due to individual difference.

For membranous structure, the thickness of membranes is generally ranging between several micrometers to dozens of micrometers, which is smaller or in the same range of micro-CT resolution $(18 \mathrm{um})$. Therefore, it makes no sense to compare the thickness of membranous structure. Instead, the measurement is made along the membranous surface. RWM has irregular shape, therefore the diameter is measure as the distance over the surface. Short diameter and long diameter of the RWM are $2.18 \mathrm{~mm}$ and $2.23 \mathrm{~mm}$ respectively, which are in line with the study performed by Nomura [15]. In his study, the short diameter of the round window is ranging from 1.43 to $2.38 \mathrm{~mm}$ (average $1.87 \mathrm{~mm}$ ) and the long diameter is ranging from 1.83 to $3.25 \mathrm{~mm}$ (average $2.3 \mathrm{~mm}$ ).

Therefore, it can be concluded that the reconstructed model reflects the real structure of the middle and inner ear. It provides reliable geometric information for anatomical structures. This model could be used for planning surgery and as well as student education in the field of otology and audiology. Furthermore, a FEM model could be generated from this model after proper modification and meshing. Application of this model could be extended to the fields of CAE and CAS.

This work is performed under the project of PBS3/B7/25/2015 founded by NCBiR.

\section{References}

1. Global Burden of Disease Study 2013 Collaborators, Lancet. 386, 743 (2015)

2. K. Jahnke, Middle Ear Surgery (Thieme, StuttgartNew York, 2004)

3. M. Neudert, T. Zahnert, N. Lasurashvili, M. Bornitz, Z. Lavcheva, C. Offergeld, Otol. Neurotol. 30, 332 (2009)

4. Y. Song, C. Lee, Tzu Chi Medical Journal, 24, 178 (2012)

5. R. Gan, C. Dai, X. Wang, D. Nakmali, M. Wood, Hearing Research, 263, 138 (2010)

6. D. Kelly, P. Prendergast, A. Blayney, Otol. Neurotol. 24, 11 (2003)

7. M. Kwacz, J. Wysocki, P. Krakowian, Biocybern Biomed Eng, 32, 27 (2012)

8. F. Dammann, A. Bode, E. Schwaderer, M. Schaich, M. Heuschmid, M. Maassen, Radio Graphics, 21, 183 (2001)

9. Y. Zhao, B. Dawant, R. Labadie, J. Noble, MICCAI 2014, Part I, 331

10. A. Paolis, H. Watanabe, J. Nelson, M. Bikson, M. Packer, L. Cardoso, J Biomech, 50, 209 (2017)

11. W. Funnell, W. Decraemer, S. Khanna, J. Acoust. Soc. Am. 91, 2082 (1992)

12. J. Lane, R. Witte, O. Henson, C. Driscoll, R. Robb, Clin Anat. 18, 409 (2015) 
13. E. Erixon, H. Hogstorp, K. Wadin, Otol Neurotol, 30, 14 (2009)

14. L. Aristeguieta, L. Acuna, Int J Morphol, 28, 1059 (2010)
15. Y. Nomura, Advances in Oto-Rhino-Laryngology, 33, 1 (1984) 